Case Report

\title{
Magnetic Attachment Retained Complete Overdenture as Treatment for Maintaning Alveolar Ridge Height - A case report
}

\author{
Anita Angdrijono, Nike Herdijantini, Hanoem Eka \\ Department of Prosthodontics, Faculty of Dental Medicine \\ Universitas Airlangga \\ Surabaya - Indonesia
}

\begin{abstract}
Background: Magnetic attachment can be used as a retentive devices in overdenture technique in prostheses. The magnets are attached with remaining root structure transfer the occlusal load to the bone through the periodontal ligament of retained roots and then prevent resorption of the alveolar ridge. Alvelar ridge plays an important role for the retention and stability of the denture as they will support the denture base. Magnetic assembly consist of magnet and coping with a keeper on the remaining tooth structure since magnetic attachment can provide support, stability and retention. Purpose: The purpose of this case report was to report magnetic attachment retained complete overdenture design and fabrication as a preserve to alveolar bone height to achieve retention and stability of the denture. Case: A 69 years old female patient, a baker and private English teacher with partial edentulous ridge came to Dental Hospital of Airlangga University asked for dentures. Clinical examination shown a complete edentulous in the upper jaw and partial edentulous in the lower jaw. The remaining teeth were 33, 34, 35, 43 and 44. 33 and 35 are chronic gangrene radix, 35 shown a slightly mobility. 34 non vital, 44 and 45 are vital with moderate resorption. Case management : All the left teeth in the lower jaw were consulted for endodontic treatment. Magnetic attachment retained complete overdenture was choosen for the lower jaw and complete denture for the upper jaw. The remaining teeth in the lower jaw kept the alveolar ridge from resorption and the magnet provided extra retention for the complete overdenture. Discussion: Overdentures supported by magnet attachment achieved greater satisfaction, gives better retention and stability for the denture. On the other side, the natural abutment teeth in overdentures preserved better proprioception and psychologically beneficial as the patient had not undergone extraction. As a conclusion, overdenture supported by magnetic attachment can be used to increase support, retention and stability especially in the lower jaw and also prevent further alveolar ridge resorption. Conclusion: Overdenture supported by magnetic attachment can be used to increase retention and the residual root also prevent further alveolar ridge resorption.
\end{abstract}

Keywords: Magnetic attachment, overdenture, edentulous

Correspondence: Nike Hendrijantini, Department of Prosthodontics, Faculty of Dental Medicine, Universitas Airlangga. J1. Prof. Dr. Moestopo No. 47 Surabaya 60132 - Indonesia. Phone: +62315030255 Email: nikeh@fkg.unair.ac.id

\section{INTRODUCTION}

The basic overdenture concept requires preservation of residual soft and hard tissue ${ }^{1}$. Use of attachment and adherence to basic principles of complete denture design can improve both retention and stability of overdenture. Traditionally, the vast majority of problems arise with a mandibular prosthesis, as due to the anatomy of the mandible often they fail to provide adequate support, retention and stability ${ }^{2}$. In attempt to these patients, a variety of aids and materials have been tried such as adhesives, and magnets.

Every clinician is aware that the proportions of the residual ridge are critical to denture success, and so it is vital to preserve the dimensions of the post extraction ridge. Magnetic attachment is a retentive appliance of removable prostheses. ${ }^{3}$ Conventional overdenture placement involves embedding the magnetic assembly in the denture base and inserting its corresponding keeper into the abutment root. The magnetic assembly holds the keeper with a retentive force. ${ }^{4}$

The residual roots are positively indicated for retention of dentures by using magnetic attachments. Until now, the aim of maintenance of residual roots was mostly for prevention of resorption of the alveolar bone which support the denture bases. By application of magnetic attachments, residual 
Indonesian Journal of Dental Medicine

Volume 1 Issue 1 2018; 54-58

roots came to play part for retentive effects as well as support of denture. ${ }^{3}$

This clinical report describes the fabrication of mandibular overdenture retained by magnets to highlight its benefits and maxillary conventional removable complete denture to rehabilitate the patient.

\section{CASE(S)}

A 69 years old female patient, a baker and private English teacher with partial edentulous ridge came to visit the Prosthodontic clinic Airlangga University Dental Hospital asked for denture. Almost all of her tooth lost due to caries that lead to extraction, last extraction were performed one month before.

Clinical examination shown a complete edentulous in the upper jaw and partial edentulous in the lower jaw. There were 5 teeth remained in the lower jaw. The remaining teeth were $33,34,35,43$ and 44.33 and 35 are chronic gangrene radix. Tooth 35 shown a slightly mobility. 34 were non vital with caries lesion, 44 and 45 are vital with gingival recesion (Figure 1).

From radiographic examination appears that 3334354344 has moderate resorption and 35 with periapical lession. 33 and 35 was gangrene radix (Figure 2). Patient was made aware of the clinical condition and she was willing to preserve the remaining teeth as long as possible.

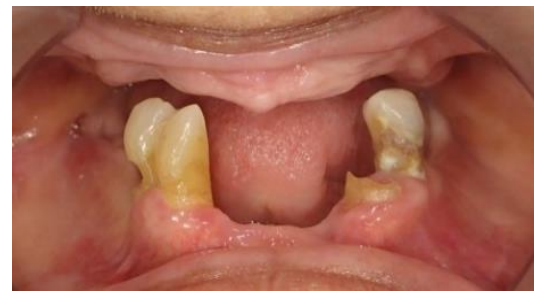

Figure 1. Intra oral anterior view.

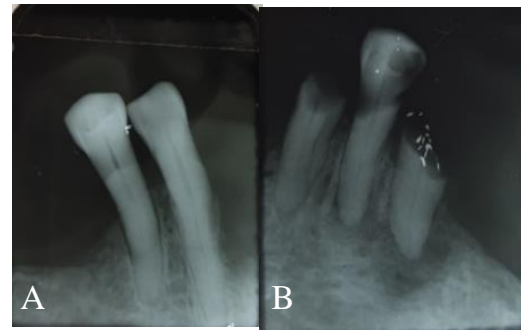

Figure 2. Panoramic radiograph. (A) tooth 44,45 (B) $33,34,35$

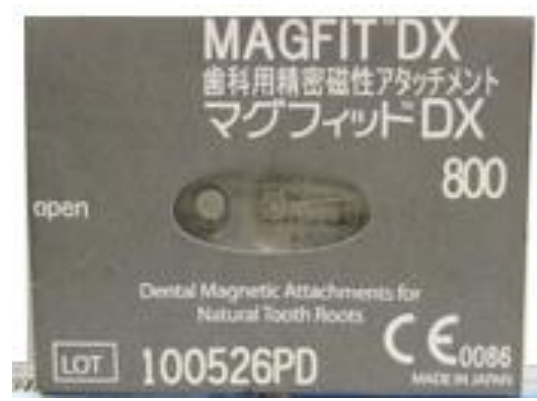

Figure 3. Magfit DX 400 magnet

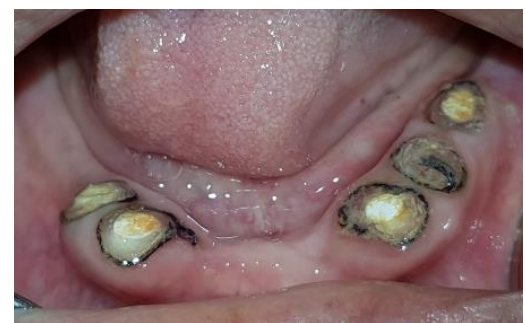

Figure 4. Decaputated and preparation on 33, 34, 35,43 and 44 with retraction cord

\section{CASE MANAGEMENT(S)}

On the basis of the clinical and radiographic examination, the different treatment options possible for this patient were extraction of remaining teeth followed by conventional complete denture, cast partial denture with or without attachments or tooth supported overdenture with or without attachments.

The case was planned to receive tooth supported overdenture using magnets. All the remaining teeth $33 \quad 34 \quad 35 \quad 43 \quad 44$ have undergone root canal treatment. The location of the remaining teeth was favourable for an overdenture. A diagnostic jaw relation was taken to analyze the amount of space available for any attachment. The space was found to be sufficient for using magnetic attachments.

Primary impression of the maxillary and mandibullar arch was made using irreversible hydrocolloid. Individual tray for was fabricated for both arch. After confirming from the patient about the treatment options, it was decided to use the remaining teeth as abutments and fabricate an overdenture with metal copings and magnetic attachments based on the advantages of the retention of the roots.

The teeth were endodontically treated and prepared with diamond rotary instrumens, producing a chamfer margin and reduced slightly above the gingival margin, followed by removal of two-third of the root canal filling material with a rotary drill instrment to 
prepare the post space to accomodate the post along with the keeper.

The attachment selected was MAGFIT DX 400 (Figure 3) closed field type of size $3,4 \mathrm{~mm}$ $\mathrm{x} 3.9 \mathrm{~mm}$ depending on the available space. Attachments were planned on 33 and 43 and metal copings on $34,35,44$.

The abutment tooth decaputated about the same as the gingival margin (Figure 4). A bevel around the circumference was made. Surface shape of the root tooth was concave.

Post space impression was made with polyvinyl siloxane and keeper was incorporated in the pattern. $\mathrm{Ni} \mathrm{Cr}$ metal coping for 3444 and 45 and cast post with keeper magnet for 33 and 43 was fabricated. Gingival retraction followed by full arch impression to record the margin area and root post was made. The metal coping and cast posts metal containing keeper magnet were carefully cemented using Glass Ionomer cement and excess cement was carefully removed (Figure $5)$.

Border molding using medium body impression material was done for maxillary and mandibular arch. Final impression was taken with polyvinyl siloxane light body (Figure 6A-B). Both impressions were carefully poured in dental stone and final casts were obtained.

Occlusal rims were fabricated on final cast, jaw relation was done and mounting the rims on the articulator, teeth were arranged and try in was done after the approval of try in by the patient, the denture was processed using heat cure acrylic resin.

Further steps of jaw relation, articulation, arrangement of teeth, try in and fabrication of denture done by conventional method of fabrication conventional denture (Figure 7AB). The dentures were carefully trimmed and polished after retrieving from the flasks.

Dentures were inserted in patient's mouth and necessary adjusment were made. To incorporate magnets in the denture, the area on the impression surface of the mandibular denture corresponding to 33 and 43 region where magnets were need to be placed was scraped to make space for magnets. Fabrication of the magnet was done after a week of usage. The magnets were positioned on the keepers in the mouth. Resin was added to the scraped area and the mandibular denture was placed over the magnet in mouth under proper occlusion. After the resin was set, denture was removed with the magnets picked up in the mandibular denture.

Excess resin was trimmed and denture polished (Figure 8A-B). After polishing the denture was again placed intraorally and checked for comfort, occlusion and retention (Figure 9). Patient was instructed how to wear and remove the denture, denture maintenance and oral hygiene as well. Patient was recalled after 24 hours, after 3 days and after 7 days.

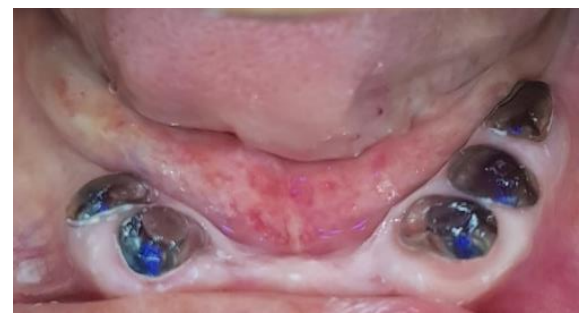

Figure 5. Cast post metal keeper magnet on 33,43 and metal coping on 34,35 and 44

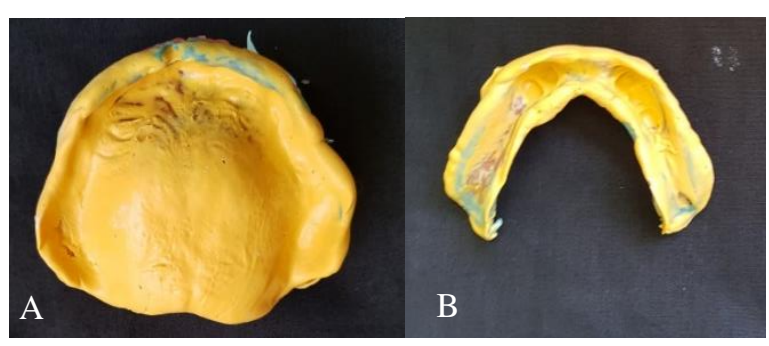

Figure 6. Final impression of maxillary arch (A) and mandibular arch (B)

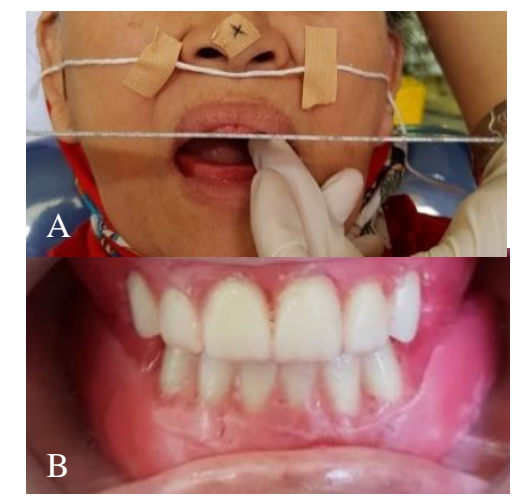

Figure 7. Taking a bite registration (A). Arrangement of the teeth (B) 


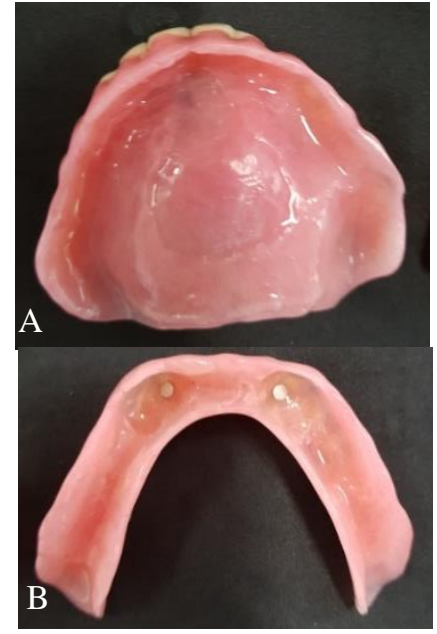

Figure 8. Magnet incorporated in (A) mandibular denture (B) maxillary denture

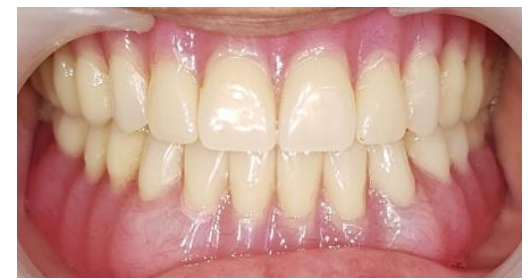

Figure 9. Anterior view of denture intraorally

\section{DISCUSSION}

Tooth loss is known problem related to oral disease, Compromised periodontal health and aging. But whenever possible extractions should be avoided as maintaining these roots or teeth aids in preservation of periodontal proprioception, reduces alveolar bone resorption. Proprioceptors of periodontal ligament provide discrete discriminating sensory input than the mucosa which helps to sense position and movement of the mandible and prevent excessive occlusal load and thus improves the masticatory performance. ${ }^{5}$

Any removable dental prosthesis that covers and rests on one or more remaining natural teeth, the roots of natural teeth, and/or dental implants, a dental prosthesis that covers and is partially supported by natural teeth, natural tooth roots, and/or dental implants is known as overdenture. This is a kind of treatment option which anchors the denture to the abutment to provide better retention, support and stability and also increase the oral health related quality of life of the patients. ${ }^{5}$

In this case, the selection of treatment for the maxillary using a complete overdenture with magnetic retention on 23 due to prominent bone and bare root metal coping on 25 , it is expected that with the retention of the magnet, the buccal flange do not need to cover the prominent bone on 23 region and it can be made open.

For mandibular using complete overdenture with bare root metal coping on 44, it is expected can improve support and denture stability, increase the supporting teeth's life expectancies, and inhibit resorption of the residual ridge of mandible. ${ }^{5}$

Overdenture with magnetic retention have the advantage of being able to increase retention, overcoming the lateral forces of the supporting tooth and easy application. ${ }^{6}$ Magnet has a small size but has a great appeal and allows placed on the bottom of the denture so as not to disturb the aesthetic especially when involving anterior teeth. ${ }^{5}$

In this case, the magnet used is an Magfit DX with 400 gf of attraction and a closedcircuit magnetic field, since the closed magnetic field has a force four times larger than the open circuit field at the same volume, where the magnetic flux density collects on the tension surface and increases magnetic flux density. ${ }^{5}$

The prognosis and use of overdenture depends on the patient's ability to perform post-insertion care, whether at home or regular visits to the dentist. Patients with overdenture should be educated about hygiene in both the oral cavity and the denture. Accumulation of plaque can reduce the effectiveness of magnetic attraction. Overdenture cleaning should be done after each meal. Patients should not use abrasive toothpaste. Special cleaning agents for denture can be used to avoid corrosion of magnets. ${ }^{7}$

Patients are encouraged to remove denture at bedtime, this is an efficient way to control the progression of periodontal disease. ${ }^{8}$ For the maintenance of denture can be by using an anti-bacterial denture cleanser, which before put into the liquid denture cleanser, the denture is cleaned first with liquid soap. When cleaning the denture it is not recommended to use toothpaste, as most contain abrasive materials that can erode acrylic resin surfaces. Patients are advised control every 6 months so prosthodontic treatment can be optimal. ${ }^{9}$

Magnetic assembly used in this case is a new generation magnetic attachment system which provides predictable retention, stability, support, and offers long term durability. Magnet retained overdenture preserving natural abutment teeth has better 


\section{Indonesian Journal of Dental Medicine}

Volume 1 Issue 1 2018; 54-58

proprioception and achieved satisfaction, and also is psychologically beneficial as the patient had not undergone extraction. ${ }^{10}$ The case showed that overdenture supported by magnetic attachment can be used to increase retention and the residual root also prevent further alveolar ridge resorption.

\section{REFERENCES}

1. D'souza, Derek. 2012. Oral Health Care Prosthodontics, Periodontology, Biology, Research and Systemic Conditions. InTech. Chapt. 2. p.19-21

2. Smitha, K \& Smintha, GP. 2015. Alveolar exostosis - revisted : A narrative review of the literature. The Saudi J for Dent. Resc. (6) : 2

3. Ai M and Shiau YY, 2004. New Magnetic Application in Clinical Dentistry. Quintessence Publ Co. Ltd. Tokyo: p. 28-69

4. Maeda Y, Nakao K, Yagi K, Matsuda S. 2006. Composite resin root coping with a keeper for magnetic attachment for replacing the missing coronal portion of a removable partial denture abutment. J Prosthet Dent;96:139-42

5. Patel , RN., Patel , SS., Sethuraman , R., Chhabra, T., Bhatt, N. 2015. Magnet retained Overdenture : A case report. Sch. J. Dent. Sci., 2(1): $1-5$

6. Rilley MA, Anthony DW, Ivor RH. 2001. Magnet in Prosthetic Dentistry. J Prosthet Dent. 86 (2) : 137-42

7. Hong,L., Ettinger, R.L., Watkins C.A., Wefel,J.S. 2003. In Vitro evaluation of fluoride varnish on overdenture abutments. J Prosthet Dent. 89(1) :28-35

8. Watt, DM, McGregor, AR. 1992, Membuat Desain Gigi Tiruan Lengkap (Designing Complete Denture). $2^{\text {nd }}$ Ed. Penerbit Hipokrates. Jakarta.

9. Zarb GA, Bolender CL, Eckert SE, Fenton AH, Jacob RF, 2004. Prostodontics treatment for edentulous patient. $12^{\text {th }}$, CV.Mosby Co, St. Louis. P. 123-25

10. Jumber JF. 1981. An atlas of overdenture and attachment, Quintessence Publishing Co Inc, Chicago; p. 17, 20-23, 113-52. 